\title{
QUALIDADE DE VIDA APÓS SETE ANOS DO EVENTO CORONARIANO AGUDO
}

\author{
Cristiane Maria Carvalho Costa Dias*, Amanda Queiroz Lemos**, lana Verena Santana Albuquerque**, \\ Cristina Aires Brasil ${ }^{* *}$, Francisco Tiago Oliveira de Oliveira***, Luciana Bilitário Macedo**** \\ Endereço para correspondência: Cristiane Maria Carvalho Costa Dia cmccdias@bahiana.edu.br \\ * Doutora em Medicina e Sáude Humana pela Escola Bahiana de Medicina e Saúde Pública, Salvador, Bahia, Brasil. \\ ** Graduado em Fisioterapia pela Escola Bahiana de Medicina e Saúde Pública, Salvador, Bahia, Brasil. \\ *** Fisioterapeuta especialista em Terapia Intensiva, Salvador, Bahia, Brasil. \\ **** Mestre em Medicina e Sáude Humana pela Escola Bahiana de Medicina e Saúde Pública, Salvador, Bahia, Brasil.
}

\section{Resumo}

Introdução: A Síndrome Coronariana Aguda (SCA) é responsável por impacto físico e psicoemocional. É apontada como uma das principais síndromes que contribuem para o prejuízo da Qualidade de Vida (QV). Estudos comprovam que as modificações dos hábitos de vida, controle de fatores de risco e a prática de atividade física causam impacto na QV destes indivíduos, tornando-se ao longo dos anos um desfecho relevante. Objetivo: Verificar a QV de indivíduos após sete anos do Evento Coronariano Agudo. Metodologia: Trata- se de um estudo transversal, composto por indivíduos diagnosticados com SCA e internados no período de abril de 2006 a janeiro de 2007 na Unidade de Terapia Intensiva Cardíaca de um hospital privado em Salvador. Foi realizado entrevista e aplicado o questionário EuroQol 5D, por contato telefônico. Resultados: Foram coletados 30 participantes, com idade média de 66,9 $\pm 11,1$ anos, predominantemente do sexo masculino $17(56,7 \%)$. Houve variação de $\% \%$ a $100 \%$ no escore total do questionário EuroQol $5 \mathrm{D}$, apresentando média de 68,2\%. Quanto aos domínios, em relação ao nível dois (problemas moderados) houve maior frequência dos domínios: Mobilidade 11 (36,7\%), Dor/mal estar 13 (19,4\%) e Ansiedade/depressão 12 (17,4\%). Enquanto no nível três (problemas extremos) foi observado: Ansiedade/depressão seis (9\%) e Dor/mal estar três $(4,5 \%)$. Conclusão: Conclui-se que há nesta população um impacto na qualidade de vida, principalmente nos domínios ansiedade/depressão e dor/mal estar após sete anos do evento agudo. A elevada taxa de fatores de risco pode estar associada a maiores escores nos domínios avaliados.

Palavras-chave: Qualidade de Vida; Síndrome Coronariana Aguda; Fatores de risco. 


\title{
QUALITY OF LIFE AFTER SEVEN YEARS OF ACUTE CORONARY EVENT
}

\begin{abstract}
Introduction: Acute Coronary Syndrome (ACS) is responsible for physical and psycho-emotional impact. It is considered one of the main syndrome that contributes to the loss of quality of life (QOL). Studies show that the changes in lifestyle, risk factor control and physical activity impact the QOL of these individuals, becoming over the years a relevant outcome. Objective: To assess the QoL of individuals after seven years of Acute Coronary Event. Methodology: This is a cross-sectional study, composed of individuals diagnosed with ACS and hospitalized from April 2006 to January 2007 in Cardiac Intensive Care Unit of a private hospital in Salvador. We conducted interviews and applied the EuroQol 5D questionnaire by telephone. Results: We collected 30 participants with a mean age of $66.9 \pm 11.1$ years, predominantly male $17(56.7 \%)$. There was variation from $0 \%$ to $100 \%$ in the total score of the EuroQol $5 \mathrm{D}$ questionnaire, with a mean of $68.2 \%$. For the domains, from the level two (moderate problems) there was a higher frequency of levels: Mobility 11 (36.7\%), pain/discomfort $13(19.4 \%)$ and anxiety/depression 12 (17.4\%). While at level three (extreme problems) was observed: Anxiety/depression six (9\%) and pain/discomfort three (4.5\%). Conclusion: We conclude that in this population there is an impact on quality of life, especially in the domains anxiety/depression and pain/ discomfort after seven years of the acute event. The high rate of risk factors may be associated with higher scores in the domains evaluated.
\end{abstract}

Keywords: Quality of Life; Acute Coronary Syndrome; Risk factors.

\section{INTRODUÇÃO}

No ano de 2010, as doenças do aparelho circulatório eram a terceira causa de internação hospitalar, representando 210.046 internações por doença isquêmica do coração, sendo esta, responsável por $29 \%$ das mortes no Brasil. (1-3) Dentre essas doenças, destaca-se a Síndrome Coronariana Aguda (SCA), que no mesmo ano registrou $7,05 \%$ do total de óbitos/ano. (2) Esta síndrome é caracterizada pela American Heart Association como um grupo de sintomas clínicos compatíveis com isquemia miocárdica aguda(4) que são ocasionadas por obstrução coronariana decorrente da interação entre fenômenos de trombose e vasoespasmo. ${ }^{(5-7)}$

Entre os clássicos fatores de risco podem-se encontrar duas categorias: os modificáveis, como o sedentarismo, ${ }^{(8)}$ hipertensão arterial sistêmica, ${ }^{(9)}$ tabagismo, dislipidemia, ${ }^{(8,10)}$ diabetes e obesida$\mathrm{de}^{(8)}$ e os não modificáveis, que são: hereditariedade, sexo e idade. (7) Torna-se necessário o controle dos fatores de risco modificáveis, uma vez que sua frequência elevada está associada à nova ocorrência de eventos cardiovasculares e impacto na Qualidade de Vida (QV). ${ }^{(1)}$ Além deste controle, preconiza-se a prática de atividade física diária, o uso regular de medicamentos e a adoção de hábitos alimentares e comportamentais saudáveis. ${ }^{(12-14)}$

Imediatamente após o evento coronariano, as condutas da equipe de saúde são instituídas ao retorno as atividades funcionais, funções físicas e estado geral de saúde desta população. ${ }^{(15)}$ É evi- 
denciado que paciente após evento coronariano agudo, durante a alta hospitalar já apresentam impacto na qualidade de vida, pincipalmente nos domínios ansiedade e depressão, ${ }^{(16)}$ portanto, a longo prazo, a QV torna-se fator importante na manutenção da saúde.

A Qualidade de Vida (QV) no contexto das cardiopatias possui relevância ao tornar-se um significativo indicador de saúde. O impacto físico e psicoemocional provocado pela doença e o alto índice de depressão têm sido apontados como os principais fatores que contribuem para o prejuízo da QV desses indivíduos ao longo dos anos. ${ }^{\left({ }^{77}\right)}$ Os indicadores de $\mathrm{QV}$ relacionados à saúde têm caráter multidimensional, para tal existem alguns instrumentos para avaliação deste aspecto, o EuroQol -5D (EQ-5D) cumpre este proposito, além de ser validado para população cardiopata ${ }^{(17,18)}$ e possuir uma aplicabilidade prática.

Dada a importância que o tema QV vem assumindo no cenário mundial, torna-se relevante a sua mensuração nesta população, considerando que com o passar dos anos pode estar associada aos fatores de risco adquiridos, devido principalmente ao envelhecimento do indivíduo. Assim uma adequada QV está vinculada ao controle dos fatores risco e adesão aos hábitos saudáveis. ${ }^{(5)}$ Neste contexto, o presente estudo tem por objetivo verificar a qualidade de vida e a frequência dos fatores de risco em indivíduos após sete anos do evento coronariano agudo.

\section{MATERIAS E MÉTODOS}

Trata-se de um estudo transversal, composto por indivíduos diagnosticados com Síndrome Coronariana Aguda e internados no período de abril de 2006 a janeiro de 2007 na Unidade de Terapia Intensiva Cardíaca de um hospital de caráter privado na cidade de Salvador, participantes de uma pesquisa prévia onde foram submetidos ao teste de caminhada de 50 metros. ${ }^{(19)}$ Foram excluídos aqueles que recusaram participar da pesquisa, in- terrompeu a entrevista, os que foram revelados por familiar e/ou cuidador com déficit cognitivo e os óbitos.

A pesquisa foi realizada por contato telefônico, no qual os participantes foram convidados a fazer parte da pesquisa, esclarecidos quanto aos objetivos da pesquisa e informados que esta poderia ser interrompida, caso despertasse recordações indesejáveis. No caso de aceitação, foi enviado o Termo de Consentimento Livre Esclarecido, por via eletrônica (e-mail). Após o consentimento, foi realizada uma breve entrevista, realizada por contato telefônico, pela pesquisadora previamente treinada, constando das seguintes perguntas: fatores de risco associados, número de internações, comorbidades, dados sócio-demográficos e hábitos de vida.

Em seguida foi aplicado o questionário de qualidade de vida, EuroQol 5D, que avalia o estado de saúde $^{(14)}$ de acordo com a percepção subjetiva do indivíduo. Este instrumento torna-se ideal para uso em pesquisas postais e entrevistas telefônicas, por precisar apenas de alguns minutos para sua aplicação. ${ }^{(20-22)}$ Este sistema compreende os seguintes domínios: mobilidade, auto-cuidado, atividades habituais, dor/desconforto e ansiedade/depressão ${ }^{(18,22)}$ para cada domínio existem três níveis de resposta: sem problemas, problemas moderados e problemas extremos. ${ }^{(18)}$ A partir destes, obtém-se um número com cinco algarismos que define um estado de saúde, dentre os 243 possíveis estados de saúde.

Após a aplicação do EQ-5D, é necessário proceder o cálculo dos índices definidos através dos cinco domínios. Este cálculo é efetuado com base nos coeficientes EQ-5D (Tabela 1), obtidos com base numa regressão do modelo desenvolvido a partir de valores recolhidos do time trade-off no Reino Unido. O termo constante é utilizado desde que exista algum problema de saúde, alguma resposta nos níveis dois (problemas moderados) e três (problemas extremos). O termo $\mathrm{N}_{3}$ é usado desde que pelo menos um domínio seja de nível três (problemas extremos). ${ }^{(20)}$ 
Tabela 1 - Coeficientes utilizados para realização do cálculo do escore de indivíduos após sete anos do Evento Coronariano Agudo submetidos ao EuroQol 5D. Salvador-Ba. 2014

\begin{tabular}{cccc}
\hline $\begin{array}{c}\text { DoMínios } \\
\text { EuroQOL 5D }\end{array}$ & COEFICIENTES \\
\cline { 2 - 4 } Mobilidade & Nível 1 & NíveL 2 & NíveL 3 \\
\hline Cuidados Pessoais & 0 & 0,069 & 0,314 \\
Atividades Habituais & 0 & 0,104 & 0,214 \\
Dor/mal-estar & 0 & 0,036 & 0,094 \\
Ansiendade/depressão & 0 & 0,123 & 0,386 \\
Constante & 0 & 0,071 & 0,236 \\
N3 & & 0,081 & \\
\hline
\end{tabular}

Sistema descritivo do EQ-5D ${ }^{23}$

O cálculo amostral foi realizado na calculadora Winpepi, utilizando como base o artigo de Ferreira 2013, ${ }^{(23)}$ utilizando como comando a estimativa de proporção para o domínio de ansiedade/depressão do EuroQol, assumindo uma proporção de $68 \%$ com uma diferença aceitável de 16 , um nível de confiança de $95 \%$, sendo necessário um número de 33 indivíduos.

A análise descritiva e analítica dos dados foi realizada através do software Statistical Package for Social Sciences (SPSS), versão 14.0 for Windows. Realizada análise de normalidade das variáveis contínuas pelo teste de Kolmogorov-Smirnov. Os resultados foram apresentados através de tabelas e figuras e as variáveis categóricas expressas em fre- quência (\%). A análise das variáveis contínuas com distribuição normal foi expressa em média e desvio

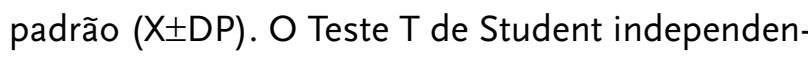
te para comparação entre grupos e as variáveis categóricas foram comparadas pelo teste Qui Quadrado, estabelecido valor de $p \leq 0,05$. As variáveis independentes estudadas foram SCA, idade, sexo, nível de atividade física, tabagismo, hábitos de vida e comorbidades, e as variáveis dependentes: a qualidade de vida mensurada através da aplicação do questionário EuroQol $5 D$.

O presente estudo obteve aprovação pelo Comitê de Ética e Pesquisa da Escola Bahiana de Medicina e Saúde Pública (EBMSP), através do CAAE: 05874112.9 .0000 .5544 . 


\section{RESULTADOS}

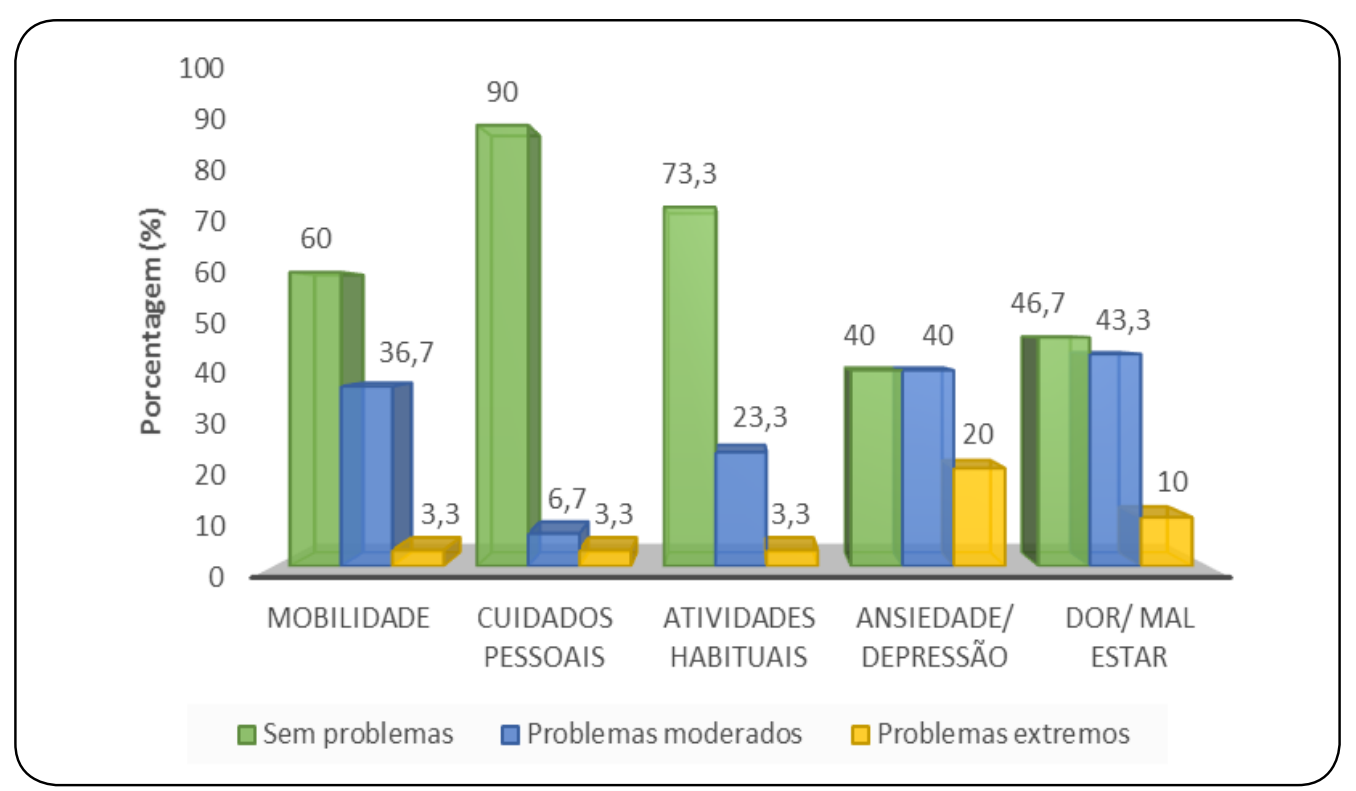

Figura 1 - Frequência dos domínios do EuroQol 5D de indivíduos após sete anos do Evento Coronariano Agudo. Salvador-Ba, 2014

Foram coletados 43 participantes e excluídos 9 indivíduos, dois $(4,4 \%)$ devido a déficit cognitivo por óbito. Cinco indivíduos $(10,9 \%)$ recusaram-se a participar da pesquisa. A amostra final foi composta por 30 participantes, destes $10(33,3 \%)$ sofreram IAM S/SST, cinco (16,6\%) IAM C/SST e 15 (50\%) Angina instável (AI) há sete anos. Houve predominância do sexo masculino, com 17 (56,7\%). A média de idade atual da população foi de $66,9 \pm$ 11,1 anos (variação de 45 a 101 anos), sendo que 20 $(66,7 \%)$ indivíduos estão inativos economicamente. Quanto às variáveis antropométricas, o índice de massa corpórea (IMC) variou de 18, o1 a 38,3 Kg/ $\mathrm{m}^{2}$, com média de $27,04 \pm 5,11 \mathrm{Kg} / \mathrm{m}^{2}$.
O resultado do EQ-5D na amostra estudada variou de $\% \%$ a $100 \%$, apresentando frequência média de $68,2 \%$ no escore total. Na população nove (30\%) indivíduos atribuíram o índice de escore 100\%, correspondente ao score máximo ED-5D. Quando analisadas as classes da SCA, observou-se que a frequência média do escore geral do EQ-5D dos indivíduos que sofreram IAM foi de $64,7 \%$ e os que apresentaram $A I$ foi de $71,7 \%(p=0,56)$, mostrando que, independente da classe da SCA, o indivíduo pode apresentar impacto na qualidade de vida. A Figura 1 apresenta a descrição dos domínios do questionário de Qualidade de Vida Euroqol-5D, e mostra que os problemas mais graves têm maior frequência no domínio ansiedade/depressão.

Tabela 2 - Fatores de Risco, características clínicas e número de internações de indivíduos após sete anos do Evento Coronariano Agudo submetidos ao EuroQol 5D. Salvador-Ba, 2014

\begin{tabular}{cc}
\hline FATORES DE RISCO & $\mathrm{N}(\%)$ \\
\hline Hipertensão & $24(80,0)$ \\
Dislipidemia & $21(70,0)$ \\
Sobrepeso/Obesidade & $19(63,3)$ \\
\hline
\end{tabular}


Tabela 2 - Fatores de Risco, características clínicas e número de internações de indivíduos após sete anos do Evento Coronariano Agudo submetidos ao EuroQol 5D. Salvador-Ba, 2014

\begin{tabular}{cc}
\hline FATORES DE RISCO & $\mathrm{N}(\%)$ \\
\hline Sedentarismo & $19(63,3)$ \\
Diabetes & $14(46,6)$ \\
Tabagismo & $\mathrm{O} 4(13,3)$ \\
Comorbidades & \\
Doença Renal & $\mathrm{O}(13,3)$ \\
DCV & $\mathrm{O3}(10,0)$ \\
Cirurgia Cardíaca & \\
Não & $22(70,0)$ \\
Sim & $\mathrm{O}(30,0)$ \\
Número de internações & $15(50,0)$ \\
Nenhuma & $10(33,3)$ \\
Uma a duas & O3 $(10,0)$ \\
Três a quatro & O2 $(6,7)$ \\
Acima de Cinco &
\end{tabular}

$\mathrm{n}(\%)=$ número de indivíduos; DCV=Doença cérebro vascular

Quanto aos fatores de risco, destacam-se a HAS 24 (80\%), Dislipidemia 21 (70\%), o sobrepeso/ obesidade e sedentarismo, ambos com 19 (63,3\%), Diabetes Melitus $14(46,7 \%)$ e quatro $(13,3 \%)$ tabagistas. Quanto ao número de internações e ao tratamento cirúrgico realizado, 15 (50\%) não tiveram internação por problema cardíaco e 22 (70\%) não foram submetidos à cirurgia cardíaca após o evento coronariano, porém, cinco $(16,7 \%)$ indivíduos realizaram angioplastia ao longo dos anos, Tabela 2. Dos indivíduos que referem episódios de precordialgia, cinco $(16,7 \%)$ referiram quando expostas ao esforço físico, e nenhum dos participantes mencionou dor ao repouso.

No que se refere aos hábitos de vida desta população e ao cumprimento das orientações da equipe multiprofissional após o evento agudo, 23 $(76,7 \%)$ dos indivíduos relataram não ingerir be- bida alcóolica, 22 (73,3\%) adota uma alimentação adequada, contudo, 19 (63,3\%) referem não pratica atividade física. Quando realizada a associação dos domínios do EQ-5D com fatores de risco, apresenta significância estatística no domínio dor/mal estar com precordialgia $(\mathrm{p}=\mathrm{O}, \mathrm{O} 2)$ e $\mathrm{o}$ tabagismo $(p=0,04)$, Tabela 3.

Foi observado que os participantes que não apresentaram a precordialgia e não tinha hábitos de fumar não relataram problemas no questionário EQ-5D. Quanto ao domínio ansiedade/depressão, houve resultado significativo quando associado ao tabagismo $(p=0,03)$, hipertensão $(p=0,03)$ e ao número de internação $(p=0,01)$. Infere-se que indivíduos hipertensos apresentam problemas moderados e extremos enquanto os que não fumam e não foram internados não apresentaram alteração no EQ-5D, Tabela 3. 
Tabela 3 - Associação entre os domínios Dor/Mal estar e Ansiedade/Depressão do EuroQol-5D com os fatores de risco, hábitos de vida e percordialgia em indivíduos após sete anos do Evento Coronariano Agudo submetidos ao EuroQol 5D. Salvador-Ba, 2014

\begin{tabular}{|c|c|c|c|c|c|c|}
\hline DoMínIOS & VAS & VEIS & NÍVEL I N(\%) & NíveL 2 N(\%) & NíveL 3 N(\%) & $P$ \\
\hline \multirow{4}{*}{$\begin{array}{c}\text { DOR/MAL } \\
\text { ESTAR }\end{array}$} & \multirow{2}{*}{ Precordialgia } & Sim & $\mathrm{O}(\mathrm{O}, \mathrm{O})$ & O5 $(38,5)$ & $\mathrm{OO}(\mathrm{O}, \mathrm{O})$ & \multirow{2}{*}{$\mathrm{O}, \mathrm{O} 2$} \\
\hline & & Não & $14(100)$ & $\mathrm{O} 8(61,5)$ & O3 (100) & \\
\hline & \multirow{2}{*}{ Tabagismo } & Sim & $\mathrm{OO}(\mathrm{O}, \mathrm{O})$ & $04(30,8)$ & $\mathrm{OO}(\mathrm{O}, \mathrm{O})$ & \multirow{2}{*}{0,04} \\
\hline & & Não & $14(100)$ & $9(69,2)$ & $03(100)$ & \\
\hline \multirow{8}{*}{$\begin{array}{l}\text { ANSIEDADE/ } \\
\text { DEPRESSÃO }\end{array}$} & \multirow{2}{*}{ Tabagismo } & Sim & $\mathrm{OO}(\mathrm{O}, \mathrm{O})$ & O4 (100) & $\mathrm{OO}(\mathrm{O}, \mathrm{O})$ & \multirow{2}{*}{0,03} \\
\hline & & Não & $12(46,2)$ & O8 $(30,8)$ & O6 $(23,1)$ & \\
\hline & \multirow{2}{*}{ Hipertensão } & Sim & $08(66,7)$ & $10(83,3)$ & O6 (100) & \multirow{2}{*}{0,03} \\
\hline & & Não & O4 $(33,3)$ & O2 $(16,7)$ & $\mathrm{OO}(\mathrm{O}, \mathrm{O})$ & \\
\hline & \multirow{4}{*}{ Internação } & Nenhuma vez & O9 $(75,0)$ & O5 $(41,7)$ & Ol $(16,7)$ & \multirow{4}{*}{0,01} \\
\hline & & Uma a duas & $\mathrm{O} 3(25, \mathrm{O})$ & $\mathrm{O} 4(33,3)$ & $\mathrm{O} 3(5 \mathrm{O}, \mathrm{O})$ & \\
\hline & & Três a quatro & $\mathrm{OO}(\mathrm{O}, \mathrm{O})$ & $\mathrm{O} 3(25, \mathrm{O})$ & $\mathrm{OO}(\mathrm{O}, \mathrm{O})$ & \\
\hline & & $>$ cinco & $\mathrm{OO}(0, \mathrm{O})$ & $\mathrm{OO}(0, \mathrm{O})$ & O2 $(33,3)$ & \\
\hline
\end{tabular}

Test qui-quadrado.

Quando realizada análise do domínio mobilidade com as demais variáveis, foi revelado significância estatística em atividade física $(p=0,03)$, dislipidemia $(p=0,01)$, número de internação $(p=0,02)$ e diabetes melitos $(p=0,01)$. Referente ao domínio atividades habituais, houve significância estatística nas variáveis atividade física $(\mathrm{p}=0,04)$, hipertensão $(p=0,05)$ e número de internação $(p=0,01)$. Observa-se que a maior parte dos participantes, que referiram sem problemas (nível um) nos domínios mobilidade e atividades habituais, não possuíam fatores de risco associados, Tabela 4.

Tabela 4 - Associação entre os domínios Mobilidade e Atividade Habituais do EuroQol-5D com os fatores de risco e hábitos de vida em indivíduos após sete anos do Evento Coronariano Agudo submetidos ao EuroQol 5D. Salvador-Ba, 2014

\begin{tabular}{|c|c|c|c|c|c|c|}
\hline DoMínIOS & \multicolumn{2}{|c|}{ VARIÁVEIS } & NíVEL I N(\%) & NíveL 2 N(\%) & NíVEL 3 N(\%) & $P$ \\
\hline \multirow{10}{*}{ Mobilidade } & \multirow{2}{*}{$\begin{array}{l}\text { Atividade } \\
\text { Física }\end{array}$} & Ativo & $10(55,6)$ & Ol $(9,1)$ & $\mathrm{OO}(\mathrm{O}, \mathrm{O})$ & \multirow{2}{*}{0,03} \\
\hline & & Sedentário & O8 $(44,4)$ & $10(90,9)$ & Ol (100) & \\
\hline & \multirow{2}{*}{ Dislipidemia } & Sim & $10(55,6)$ & $11(100)$ & $\mathrm{OO}(\mathrm{O}, \mathrm{O})$ & \multirow{2}{*}{0,01} \\
\hline & & Não & O8 $(44,4)$ & $\mathrm{OO}(0, \mathrm{O})$ & Ol (100) & \\
\hline & \multirow{4}{*}{ Internação } & Nenhuma vez & $12(66,7)$ & O3 $(27,3)$ & $\mathrm{OO}(\mathrm{O}, \mathrm{O})$ & \multirow{4}{*}{$\mathrm{O}, \mathrm{O} 2$} \\
\hline & & Uma a duas & O5 $(27,8)$ & O5 $(45,5)$ & $\mathrm{OO}(\mathrm{O}, \mathrm{O})$ & \\
\hline & & Três a quatro & $\mathrm{OO}(\mathrm{O}, \mathrm{O})$ & O2 $(18,2)$ & Ol (100) & \\
\hline & & $>$ cinco & $\mathrm{Ol}(5,6)$ & Ol $(5,6)$ & $\mathrm{OO}(\mathrm{O}, \mathrm{O})$ & \\
\hline & \multirow{2}{*}{ Diabetes } & Sim & O5 $(27,8)$ & О9 $(81,8)$ & $\mathrm{OO}(0,0)$ & \multirow{2}{*}{0,01} \\
\hline & & Não & $13(72,2)$ & O2 $(18,2)$ & Ol (100) & \\
\hline
\end{tabular}


Tabela 4 - Associação entre os domínios Mobilidade e Atividade Habituais do EuroQol-5D com os fatores de risco e hábitos de vida em indivíduos após sete anos do Evento Coronariano Agudo submetidos ao EuroQol 5D. Salvador-Ba, 2014

(conclusão)

\begin{tabular}{|c|c|c|c|c|c|c|}
\hline DoMínIOS & \multicolumn{2}{|c|}{ VARIÁVEIS } & NíVEL I N(\%) & NíVEL 2 N(\%) & Nível 3 N(\%) & $P$ \\
\hline \multirow{6}{*}{$\begin{array}{l}\text { Atividades } \\
\text { Habituais }\end{array}$} & Atividade & Ativo & $11(50,0)$ & $\mathrm{OO}(\mathrm{O}, \mathrm{O})$ & $\mathrm{OO}(\mathrm{O}, \mathrm{O})$ & $\cap$ \\
\hline & Física & Sedentário & $11(50,0)$ & 07 (100) & Ol (100) & 0,04 \\
\hline & \multirow{4}{*}{ Internação } & Nenhuma vez & $13(59,1)$ & O2 $(28,6)$ & $\mathrm{OO}(\mathrm{O}, \mathrm{O})$ & \multirow{4}{*}{0,01} \\
\hline & & Uma a duas & O5 $(22,7)$ & O5 $(71,4)$ & $\mathrm{OO}(\mathrm{O}, \mathrm{O})$ & \\
\hline & & Três a quatro & O2 $(9,1)$ & $\mathrm{OO}(\mathrm{O}, \mathrm{O})$ & Ol (100) & \\
\hline & & $>$ cinco & O2 $(9,1)$ & $\mathrm{OO}(\mathrm{O}, \mathrm{O})$ & $\mathrm{OO}(\mathrm{O}, \mathrm{O})$ & \\
\hline
\end{tabular}

Test qui-quadrado.

\section{DISCUSSÃO}

A mensuração da QV tem se tornado cada vez mais relevante na área de saúde, por ser considerada um fator importante na análise do impacto de um tratamento frente a uma doença crônica, ${ }^{(24)}$ porém esta deve valorizar a perspectiva do sujeito em relação às várias dimensões de sua vida e não só em relação à intensidade de sintomas. Embora estudos abordem a QV de indivíduos após evento coronariano, ${ }^{(25)}$ em sua maioria, eles buscam avaliar o resultado de intervenções, ${ }^{24,26,27)}$ havendo uma escassez quando analisada a longo prazo. O presente estudo revelou que os domínios ansiedade/ depressão e dor/mal estar são os que apresentam maior comprometimento.

De acordo com Gorenstein e cols. (1999) ${ }^{(28)}$ e Oliveira JR (2005) ${ }^{(29)}$ indivíduos demonstram alto grau de ansiedade após um IAM, tal dado confirma os achados deste estudo. A literatura aponta a depressão como um fator de risco tanto para o surgimento da doença coronariana quanto para uma maior morbidade e mortalidade em cardiopatas. (30,31) Para estes, o evento coronariano representa uma ameaça à morte, mostrando-se como um obstáculo para a execução das atividades de vida diária, e a forma como enfrenta a doença, impactando assim, negativamente na QV a longo prazo. (30)

Este estudo revelou que indivíduos que mencionaram problemas no domínio ansiedade/depressão, de forma moderada ou extrema, apresenta- ram também problemas no domínio mobilidade e dor/mal estar, com significância estatística. Pode-se inferir que o estado emocional, especificamente a depressão, podem acometer o sistema cardiovascular com diminuição da função vagal e aumento relativo da função simpática. A disfunção autonômica pós IAM é associada as maiores taxas de morbimortalidade, causa direta de um evento cardiovascular secundário e limitação física. ${ }^{(32-34)}$

Sardinha et al. em 2011 ressalta que a depressão e ansiedade, podem contribuir tanto para déficits funcionais, além de ser considerado fator de risco adicional. ${ }^{(35)} \mathrm{A}$ presença destes sintomas após o IAM está relacionada à maior reincidência, morte por doença cardíaca, prejuízo funcional pós infarto, maior dificuldade para aderir as dietas e em seguir orientações médicas, possuindo percepção distorcida do seu estado físico, além das dificuldades em relação às mudanças de estilo de vida, como a realização de atividade física, o que, por sua vez, justifica a importância do seu diagnóstico e tratamento precoce de forma a reduzir os efeitos deletérios potenciais de distúrbios emocionais persistentes. ${ }^{(10,19)}$ Dentre os ansiosos e/ou deprimidos, mostrar-se maior frequência de indivíduos inativos, sedentários e estiveram internados por problema cardíaco, em algum momento, durante os sete anos após o evento, corroborando com o estudo supracitado.

Quanto ao hábito de fumar, na amostra estudada, todos apresentaram problemas no domínio ansiedade/depressão após sete anos do evento co- 
ronariano, apresentando significância estatística. Estudo aponta que o nível de ansiedade dos fumantes é maior do que em não-fumantes. ${ }^{(36)}$ Contudo os indivíduos não tabagistas também relataram problemas no domínio ansiedade/depressão, esta ocorrência pode ser justificada devido às características psicológicas associadas ao cardiopata ou por causa da abstinência do cigarro, pois a nicotina produz um efeito psicoativo nos receptores colinérgicos que geram sensações prazerosas ${ }^{(36)}$ e a sua escassez poderá levar a quadros de ansiedade.

Quando relacionado, o domínio dor/mal estar do EuroQol $5 \mathrm{D}$ ao sintoma de precordialgia, foi observado maior frequência de indivíduos que não apresentavam este sintoma mencionando problemas no domínio dor/mal estar, a maior parte da amostra referiu quadros álgicos musculoesqueléticos, justifica-se este fato, através do processo de envelhecimento da população, que caracteriza-se pela alta incidência de doenças crônicas, ${ }^{(37,38)}$ aspecto este que pode interferir na qualidade de vida.

Foi observado maior frequência de HAS na população estudada, seguida pela dislipidemia e sobrepeso/obesidade, corroborando com a literatura. ${ }^{(39,40)}$ O sedentarismo também apresentou maiores frequências neste estudo, dado relevante, uma vez que a prática regular de atividade física reduz as taxas de mortalidade, contribui para a redução da ocorrência de outros eventos coronarianos, aumento da capacidade funcional, controle de fatores de risco cardiovascular, além do benefício psicológico representado pela diminuição da sensação de invalidez e aumento da autoconfiança, contribuindo para o bem-estar. ${ }^{(40)}$

Dentre os indivíduos ativos, apenas $18,2 \%$ praticam atividade física supervisionada. Recentemente, Queiroz et al. 2013, demonstrou eficácia da caminhada não supervisionada na melhora da aptidão física e redução do risco cardiovascular, destacando o efeito hipotensor deste treinamento em indivíduos hipertensos com a redução do colesterol total e massa corpórea, contudo não foi observado redução significante dos demais fatores de risco. (40) Ainda não existe evidencias deste treinamento na população pós evento coronariano, destaca-se, portanto, a necessidade de um programa supervisionado que englobe uma abordagem de intervenções múltiplas para a modificação do maior número possível de fatores de risco e o controle dos possíveis efeitos adversos durante a prática do exercício físico.

É evidente que em indivíduos coronariopatas se faz necessário um controle dos fatores de risco modificáveis, por meio das mudanças de hábitos de vida para que assim possa impedir a evolução desta doença, causando impacto na QV, influenciando na habilidade individual para desenvolver e manter as mudanças no estilo de vida, necessárias para a prevenção secundária destas afecções.

Como limitação do estudo, pode-se citar seu caráter transversal o qual impossibilitou a comparação, a fim de determinar o impacto da Qualidade de Vida após os sete anos do Evento Coronariano Agudo nesta população e a não aplicação da escala visual analógica (EVA) associada ao questionário EuroQol 5 D. Sugere-se, um desenho metodológico apropriado e o acréscimo de um grupo controle, composto por uma população hígida.

Conclui-se que há nesta população um impacto na qualidade de vida, principalmente nos domínios ansiedade/depressão e dor/mal estar após sete anos do evento agudo. A elevada taxa de fatores de risco, como HAS e sedentarismo, pode estar associada a maiores escores nos domínios avaliados.

\section{REFERÊNCIA}

1. Brasil. Ministério da Saúde. Datasus. Sistema de informações de mortalidade. Óbitos por residência por ano do óbito segundo capítulo CID-10. Dados preliminares de mortalidade 2010. [Acesso em 2013 ago]. Disponível em: http://www.datasus.gov.br

2. Piegas LS, Guimarães AAHP, Muniz AJ, Reis HJL, Santos ES. Comportamento da Síndrome Coronariana Aguda. Resultados de um Registro Brasileiro. Arq Bras Cardiol. 2013; 10O(6):5O2510. 
3. Bento VFR, Brofman PRS. Impacto da consulta de enfermagem na frequência de internações em pacientes com insuficiência cardíaca em Curitiba - Paraná. Rev. bras. cardiol. 2009;92(6):490-496.

4. American Heart Association-Learn and Live [Acesso em 2013 set]. Disponível em: www. heart.org/HEARTORG/

5. Lemos KF, Davis R, Moraes MA, Azzolin, K. Prevalência De Fatores De Risco Para Síndrome Coronariana Aguda Em Pacientes Atendidos Em Uma Emergência. Rev. gaúch. enferm. 2010; 31(1):129-35.

6. Dessotte CAM, Dantas RAS, Schmidt A. Sintomas de pacientes antes da primeira hospitalização por Síndrome Coronariana Aguda. Rev. Esc. Enferm. USP. 2011; 45(5):10971104.

7. Silva MAD, Sousa AGMR, Schargodsky, H. Fatores de Risco para Infarto do Miocárdio no Brasil: Estudos Fricas. Arq. bras. cardiol. São Paulo, 1998;71(5):667-675.

8. Araújo F, Yamada AT, Araújo MVM, Latorre MRDO, Mansur AJ. Perfil Lipídico de Indivíduos sem Cardiopatia com Sobrepeso e Obesidade. Arq. bras. cardiol. 2005; 84(5):405-409.

9. Alcântara EC, Resende ES, Paula LKG, Silveira LC, Costa MD. Avaliação da qualidade de vida após infarto agudo do miocárdio e sua correlação com o fator de risco hipertensão arterial. Rev. bras. hipertens. 2007;14(2):118-120.

1O. Alves TCTF, Fráguas R, Wajngarten M. Depressão e infarto agudo do miocárdio. Rev. psiq. clín. 2009;36(3):88-92.

11. Gallani MC, Cornélio ME, Agondi RF, Rodrigues RCM. Estrutura conceitual para pesquisa e prática clínica na mudança de comportamentos em saúde cardiovascular. Rev. latino-am. enfermagem. 2013;21(9).

12. Nery RM, Barbisan JN, Mahmud MI. Influência da prática da atividade física no resultado da cirurgia de revascularização miocárdica. Rev. bras. cir. cardiovasc. 2007;22(3):297-302.

13. Hedbäck B, Perk J, Hörnblad M, Ohlsson U. Cardiac rehabilitation after coronary artery bypass surgery: 10-years results on mortality, morbidity and readmissions to hospital. J. cardiovasc. risk. 2001;8(3):153-8.

14. Taylor RS, Brown A, Ebrahim S, Jolliffe J, Noorani H, Rees K, et al. Exercise-based rehabilitation for patients with coronary heart disease: systematic review and meta-analysis of randomized controlled trials. Am. j. med. 2004;116(10):682-92.

15. Eriksson M, Asplund K, Hochwalder J, Svedlund $M$. Changes in hope and health-related quality of life in couples following acute myocardial infarction: a quantitative longitudinal study. Scand. j. caring. sci. 2013;27;295-302. doi:10.1111/j.1471-6712.2012.01032.x.

16. Dias CMCC, Macedo LB, Gomes LTJC, Oliveira PLSP, Albuquerque IVS, Lemos $A Q$, et al. Quality of Life of Patients After an Acute Coronary Event: Hospital Discharge. J. clin. med. res. 2014. doi: http://dx.doi.org/10.1474O/ jocmr1865w.

17. Silva SA, Passos SRL, Carballo MT, Figueiró $M$ et al. Avaliação da qualidade de vida após síndrome coronariana aguda: revisão sistemática. Arq. bras. cardiol. 2011;97(6):526540.

18. Balestroni G, Omarini G, Omarini P, Zotti AM. L'EuroQoL 5D per la valutazione della qualità della vita in riabilitazione cardiologic. Supplemento B, Psicologia. 2007;29(3):B56-B62.

19. Dias CMCC, Maiato ACCA, Baqueiro KMM, Fiqueredo AMF, Rosa FW, Pitanga JO, et al. Resposta circulatória à caminhada de $50 \mathrm{~m}$ na unidade coronariana, na síndrome coronariana aguda. Arq. bras. cardiol. 2009;92(2):135-142.

2O. Calvert MJ, Freemantle N, Cleland JGF. The impacto $f$ chronic heart failure on health-related quality of life data acquired in the baseline phase of the CARE-HF study. The European Journal of Heart failure. 20057 (2005); 243251.

21. Schweikert B, Hahmann H, Leidl R. Validation of the EuroQol questionnaire in cardiac rehabilitation. Cardiovascular Medicine, 2006.

22. EuroQol Group: Cheung K, Oemar M. et al. User guide: Basic informationon how to use EQ-5D, version 2.O. March, 2009. [acesso em 2013 set]. Disponível em: www.euroqol.org 
23. Ferreia PL, Ferreira LN, Pereira LN. Contributos para a validação da versão portuguesa do EQ5D. Acta med. port. 2013;26(6):664-675.

24. Rossi RC, Vanderlei FM, Medina LAR, Pastre CM, Pandovani CR, Vanderlei LCM. Influência do perfil clínico e Sociodemográfico na Qualidade de Vida de cardiopatas submetidos à Reabilitação Cardíaca. Conscientiae saúde.2O11;10(1):59-68.

25. Lemos V, Sologurem MJJ, Sousa, TC, Cravo SL. Qualidade de vida em pacientes após infarto Agudo do miocárdio. Rev. Soc. Cardiol. Estado de São Paulo, 2003; 13(4) (supl A).

26. Campos FVS, Porto LGG. Qualidade de Vida e nível de atividade física de pacientes em fase ambulatorial da Reabilitação Cardíaca. Rev. bras. ativ. fís. saúde. 2009;14(2):86-95.

27. Takiuti ME, Hueb W, Hiscock SB, Nogueira CRSR, Girardi P, Fernandes F, et al. Qualidade de vida após revascularização cirúrgica do miocárdio, Angioplastia ou tratamento clínico. Arq. bras. cardiol. 2002;88(5):537-544.

28. Gorenstein C, Andrade HSG, Antonio LZ. Escalas de avaliação clínica em psiquiatria e psicofarmacologia. Rev. psiquiatr. clín.1999;25,89-143.

29. Oliveira Jr. W. Relação médico paciente em Cardiologia: um olhar psicossomático. Revista de Medicina Psicossomática Sul Mineira. 2005;4,41-46.

30. Favarato MECS, Favarato D, Hues WA, Aldrighi JM. Qualidade de vida em portadores de doença Arterial Coronária: Comparação entre gêneros. AMB rev. Assoc. Med. Bras. 2006;52(4): 236-41.

31. Lemos C, Gottschall CAM, Pellanda LC, Muller M. Associação entre depressão, ansiedade e Qualidade de Vida após infarto do Miocárdio. Psicol. teor. pesqui. Brasília, 2008; 24(4): 47176.

32. Kop WJ, Stein PK, Tracy RP, Barzilay JI, Schulz R, Gottdiener JS. Autonomic nervous system dysfunction and inflammation contribute to the increased cardiovascular mortality risk associated with depression. Psychosom. med. 2010;72:626-35.

33. J T Bigger Jr JT, Fleiss JL, Steinman RC, Rolnitzky LM, Kleiger RE, Rottman JN. Frequency domain measures of heart period variability and mortality after myocardial infarction. Circulation. 1992;85:164-171

34. Hillebrand S, Gast KB, de Mutsert R, Swenne CA, Jukema JW, Middeldorp S, et al. Heart rate variability and first cardiovascular event in populations without known cardiovascular disease: meta-analysis and dose-response metaregression. Europace. 2013;15(5):742-749

35. Sardinha A, Araújo CGS, Oliveira e Silva $A C$, Nardi AE. Prevalência de transtornos psiquiátricos e ansiedade relacionada à saúde em coronariopatas participantes de um programa de exercício supervisionado. Rev. psiquiatr. clín. 38(2):61;2011.

36. Serrano Jr. CV, Setani KT, Sakamoto E, Andrei $A M$, Fraguas R. Association between depression and development of coronary artery disease: pathophysiologic and diagnostic implications. Vasc. health and risk manag. 2011;7:159-164.

37. Ramos LR, Rosa TEC, Oliveira ZM, Medina MCG, Santos FRG. Perfil do idoso em área metropolitana na região sudeste do Brasil: resultados de inquérito domiciliar. Rev. saúde pública. 1993;27:87-94.

38. Moraes AS, Freitas ICM. Doença isquêmica do coração e fatores associados em adultos de Ribeirão Preto, SP. Rev. saúde pública. 2012;46(4):591-601.

39. Siviero IMPS, Scatena MCM, Junior MLC. Fatores de risco numa população de infartados. Rev. Enferm. UERJ. 2005;13:319-24.

4O. Queiroz ACC, Brito LC, Santos MA, Fecchio RY, Stocco ALB, Bezerra Al. Prescrição de atividade física não supervisionada, risco cardiovascular e aptidão física. Rev. bras. educ. fis. esp. 2013;27(3):377-86. 